\title{
Angular resonance absorption spectra of Langmuir-Blodgett films studied by the photoacoustic technique
}

\author{
Yao-Chun Shen*, Shu-Yi Zhang and Yue-Song Jiang \\ Institute of Acoustics, Nanjing University, Nanjing 210008, China
}

Rong Zhu and $\mathrm{Yu}$ Wei

Chien-Shiung $W_{u}$ Laboratory, Southeast University, Nanjing 210018, China

(Received October 5, 1993; accepted January 25, 1994)

\begin{abstract}
The photoacoustic (PA) angular spectrum method was developed on the basis of attenuated total reflection (ATR) and PA spectroscopies. This method was used to obtain the resonance absorptioin spectrum of Langmuir-Blodgett (LB) films on silicon substrates. The rigorous electromagnetic theory in a layered medium is also presented to explain the experimental results and to characterize the films. By fitting theoretical results to the experimental spectrum, the refractive indices and the thickness of the films can be evaluated. Moreover, as the intensity of the laser beam increased, additional peaks were observed in the angular PA spectrum, predicted to be related to the optical non-linearity of LB films. Compared with conventional ATR spectroscopy, PA ATR spectroscopy has the advantages of a simpler optical system and higher sensitivity, as well as a wider application range.
\end{abstract}

\section{Introduction}

Resonance attenuated total reflection (ATR) spectroscopy is a well-established method for the study of surfaces and thin overlayers. This technique has been successfully developed to characterize, non-destructively, layered structures such as an epitaxial Si [1] layer and multilayer heterostructures [2]. In the technique, a hemicylindrical prism coupler is used to excite electromagnetic resonance modes in the film so that the angular absorption spectrum presents the characteristics of resonance absorption, i.e. the sharp resonance absorption peaks appear in the spectrum. From theoretical analysis of the resonance absorption spectrum, especially of the positions of the peaks, the thickness and/or the refractive indices of the films can be determined.

In previous work, the resonance absorption spectrum has been obtained by measuring the intensity of the light reflected from the films. However, the intensity of the reflected light decreased dramatically when resonance absorption occurred; furthermore, the angle of the reflected light changes at double the angle of the incident light. Therefore the requirements of measuring the reflected light complicate the experimental set-up and also limit the application range of this technique.

\footnotetext{
* Present address: National Laboratory of Molecular and Biomolecular Electronics, Southeast University, Nanjing 210018, China.
}

In this paper, we combine the photoacoustic (PA) method [3-5] with the ATR technique in order to avoid measuring the reflected light. The PA method probes absorption of photons by directly detecting the heat production resulting from the non-radiative dissipation of the excited states created by incident photons; this approach has high sensitivity, especially for samples with low optical absorption coefficients. Therefore, the resonance absorption spectra of Langmuir-Blodgett (LB) films on silicon substrates can be obtained easily. In addition, by fitting theoretical calculations to the experimental spectra, the refractive indices and the thicknesses of the films can be evaluated. Compared with the conventional ATR technique, the PA ATR spectroscopy has the advantages of a simpler optical system, higher sensitivity and wider applicatoin potential.

\section{Experimental details}

The LB film of manganese stearate and polymerized diacetylene studied were deposited separately on silicon substrates by the conventional LB film deposition technique $[6,7]$, in which the amphiphilic molecules in two neighbouring sublayers are arranged in head-to-head and tail-to-tail mode.

PA resonance absorption spectroscopy is a combination of traditional PA and ATR spectroscopies. In the experiment, the resonance PA absorption spectrum can 


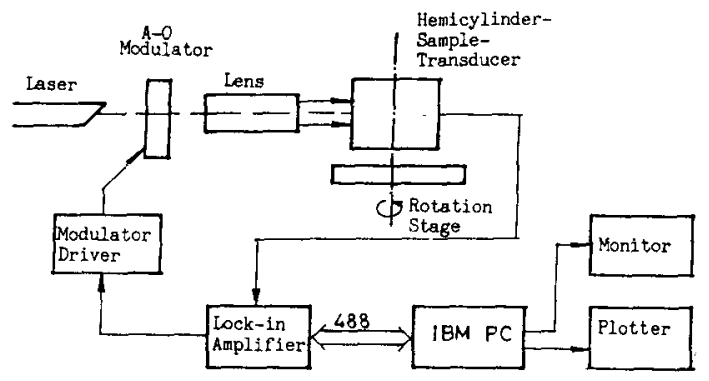

Fig. 1. Schematic diagram of the experimental set up.

be obtained by the measurement of the PA signal, either as a function of the wavelength $\lambda$ of the incident laser beam at fixed incident angle $\theta$ (i.e. the wavelengthdependent PA spectrum), or as a function of $\theta$ at fixed $\lambda$ (the angular-dependent PA spectrum). We prefer to measure the angular spectrum, which simplifies the analysis of the results by avoiding dispersion effects. Figure 1 shows a schematic diagram of the experimental set-up. A p-polarized argon ion laser with wavelength $488 \mathrm{~nm}$ modulated by an acousto-optic modulator is used to illuminate the sample. A hemicylindrical prism coupler with refractive index 1.523 is used to couple light into the LB film as shown in Fig. 2. The film acts as the optical resonance cavity layer and the substrate acts as the optical absorber. The prism coupler is placed prone on the film separated from it by an appropriate air gap which is adjusted empirically to optimize the sharpness of the PA spectrum. A cylindrical PZT (lead titanate-zirconate) piezoelectric transducer with a longitudinal vibrational mode is bonded on the back of the silicon substrate. The PA resonance spectrum can be obtained by changing the incidence angle of the laser beam by rotating the sample stage.

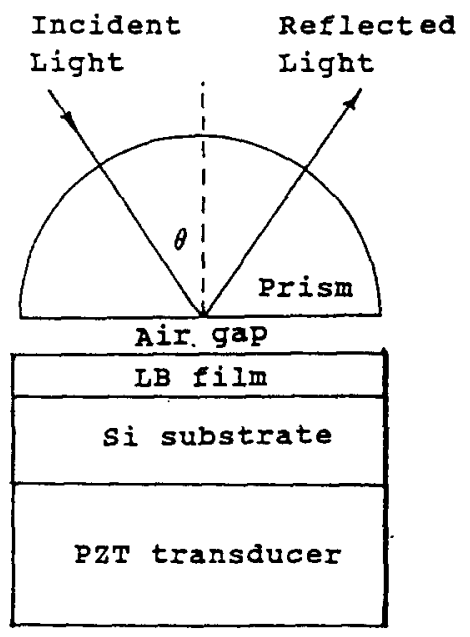

Fig. 2. Configuration of the transducer-sample-hemicylinder geometry.

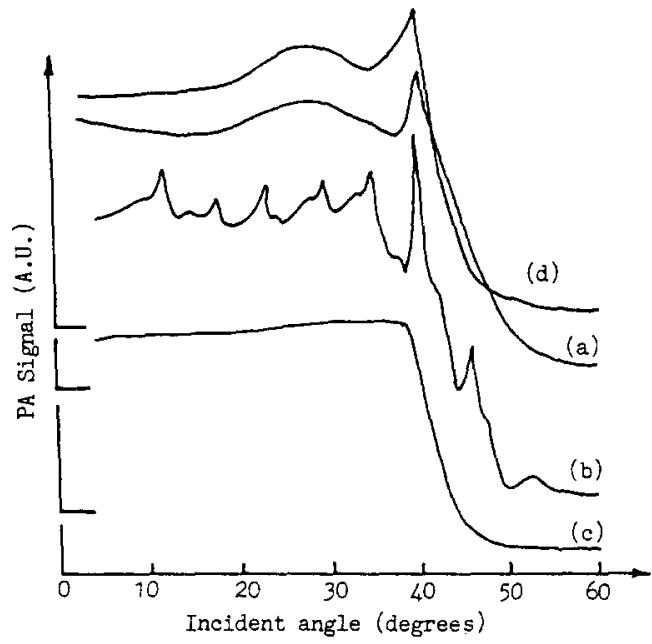

Fig. 3. Angular PA spectra: curves a-c, experimental results for LB film on the Si wafer with a planar laser beam (curve a), LB film on the Si wafer with a focused laser beam (curve b), a bare Si wafer with a focused laser beam (curve c), and theoretical results (curve d).

Curve a in Fig. 3 is the measured angular PA spectrum of manganese stearate films with 51 layers. There is an absorption peak at about $40^{\circ}$ in curve a which corresponds to the waveguide resonance mode in the $\mathrm{LB}$ film since the angular PA spectrum of the bare $\mathrm{Si}$ wafer (without LB film) displays no absorption peak (see curve $c$ of Fig. 3). The experimental results also show that the position of the peak is independent of the thickness of the air gap between sample and hemicylinder although the shape of the spectrum may change with the thickness of the air gap.

Moreover, as the pump laser power increases, additional peaks are found in the angular PA spectrum of the LB film on the Si wafer, as shown in curve b of Fig. 3. It is predicted that the additional peaks may be related to the optical non-linearity of the LB film. This can be illustrated more clearly by the following experiment. In the experiment a lens of focal length $15 \mathrm{~cm}$ is used to focus the laser beam and, by changing the distance between the sample and the focal plane, the laser intensity on the sample will be changed. Figure 4 shows the angular PA spectra of LB film on the $\mathrm{Si}$ wafer at different laser intensities, where the LB film is an assembly 51 monolayers thick of polymerized diacetylenes deposited on the $\mathrm{Si}$ substrate. It can be confirmed from Fig. 4 that the non-linearity of LB film causes the additional peaks in the angular PA spectrum.

\section{Theoretical analysis}

In order to explain the experimental results and characterize the LB films, an outline of the rigorous 


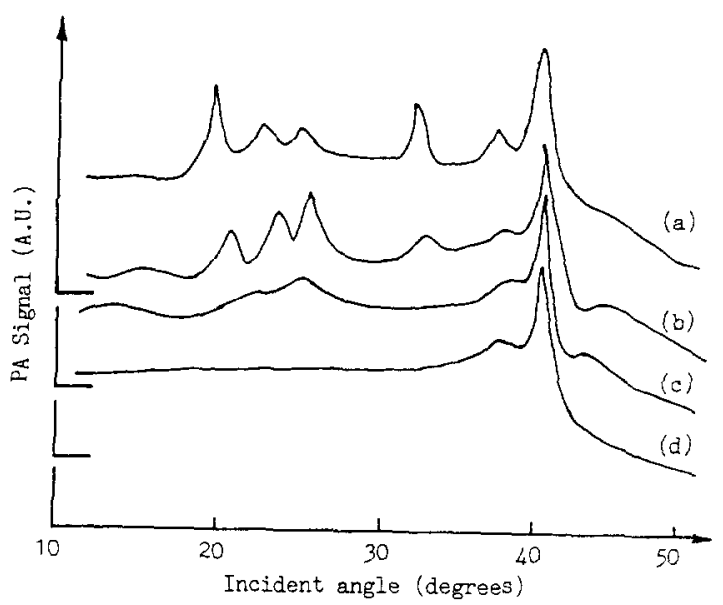

Fig. 4. Angular PA spectra of the LB film on the Si wafer at different laser intensities. The distances between sample and focal plane are 0 (curve a), $2 \mathrm{~cm}$ (curve b), $4 \mathrm{~cm}$ (curve c) and $6 \mathrm{~cm}$ (curve d).

electromagnetic theory for a layered medium, and some of the numerical results, are presented below.

\subsection{Outline of the theory}

In the theory, we assume that (i) the sample is $M$ layered in the depth direction and uniform in the transverse, and (ii) the incident light is p polarized. Thus the one-dimensional multilayer model, as shown in Fig. 5 , can be used. The $z$ component of the magnetic field, which is the only magnetic component for p-polarized light, can be expressed as

$$
\begin{aligned}
& H_{z}^{(i)}(x, y)=A_{i} \exp \left(j\left(\alpha x-\beta_{i} y\right)\right)+B_{i} \exp \left(j\left(\alpha x+\beta_{i} y\right)\right) \\
& (i=1,2, \ldots, M)
\end{aligned}
$$

where

$$
\begin{aligned}
& \alpha=\epsilon_{1}^{1 / 2} k_{0} \sin \theta \\
& \beta_{i}=\left(\epsilon_{i} k_{0}^{2}-\alpha^{2}\right)^{1 / 2}
\end{aligned}
$$

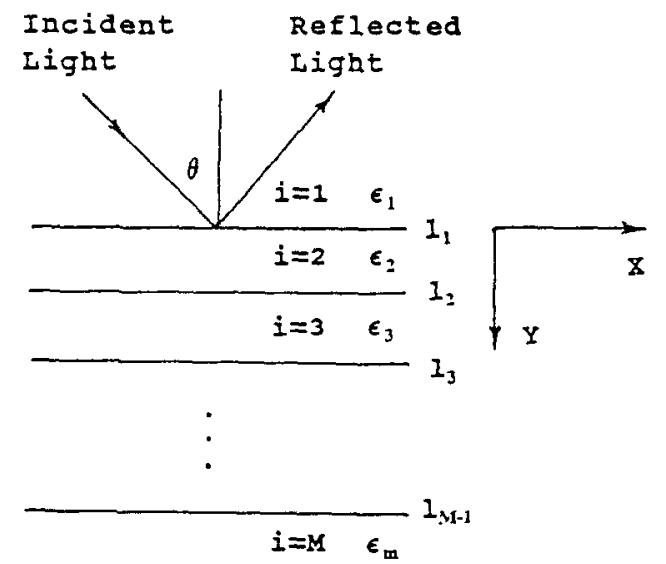

Fig. 5. One-dimensional multilayer model used in the theory. with $k_{0}$ the wavevector of light in vacuum, $\theta$ the incidence angle and $\epsilon_{i}$ the dielectric constant in the $i$ th layer of the sample. The $x$ component of the electric field can be obtained from Maxwell's equation

$E_{x}^{(i)}(x, y)=\frac{1}{j \omega \epsilon_{i}} \frac{\partial\left[H_{z}^{(i)}(x, y)\right]}{\partial y}$

According to the continuity of $H_{z}(x, y)$ and $E_{x}(x, y)$ at the boundary $y=l_{i}$, the boundary conditions are

$$
\begin{aligned}
& H_{z}^{(i)}\left(x, l_{i}\right)=H_{z}^{(i+1)}\left(x, l_{i}\right) \\
& E_{x}^{(i)}\left(x, l_{i}\right)=E_{x}^{(i+1)}\left(x, l_{i}\right) \\
& (i=1,2, \ldots, M-1)
\end{aligned}
$$

We can get the iterative formula from eqn. (4) as follows:

$$
\begin{aligned}
A_{i}= & \frac{\exp \left(j \beta_{i} l_{i}\right)}{2}\left[A_{i+1} \exp \left(-j \beta_{i+1} l_{i}\right)\left(1+\tau_{i}\right)\right. \\
& \left.+B_{i+1} \exp \left(j \beta_{i+1} l_{i}\right)\left(1-\tau_{i}\right)\right] \\
B_{i}= & \frac{\exp \left(j \beta_{i} l_{i}\right)}{2}\left[A_{i+1} \exp \left(-j \beta_{i+1} l_{i}\right)\left(1-\tau_{i}\right)\right. \\
& \left.+B_{i+1} \exp \left(j \beta_{i+1} l_{i}\right)\left(1+\tau_{i}\right)\right]
\end{aligned}
$$

where $\tau_{i}=\epsilon_{i} \beta_{i+1} / \epsilon_{i+1} \beta_{i}$. Given arbitrarily $A_{M}=1$ and $B_{M}=0, A_{1}$ and $B_{1}$ can be obtained by iterating eqn. (3). The refiectance $R$, the transmittance $T$ and absorptance $A$ can be written as

$R=\left|B_{1} / A_{1}\right|$

$T=\left|1 / A_{1}\right|$

$A=1-R-T$

In addition, for s-polarized incident light, a similar theoretical analysis shows that eqns. (4) and (5) are also suitable, but $\tau_{i}$ should be replaced by $\tau_{i}^{\prime}$, where $\tau_{i}^{\prime}=\beta_{i+1} / \beta_{i}$.

\subsection{Numerical results}

In order to evaluate the relations between the positions of the resonance absorption peaks and the parameters of the films, some simulated spectra of the films with different refractive indices and thicknesses have been calculated, as shown in Figs. 6 and 7. In the calculation, the indices of the hemicylindrical prism and the silicon substrate are 1.965 and 3.85-0.077i respectively. The incident light is s polarized with wavelength $6328 \AA$. We can see from Fig. 6 that, as the thickness of the film increases from 0.5 to $1.0 \mu \mathrm{m}$, the number of absorption peaks increases from two to four. Figure 7 shows that the angular positions of the absorption peaks increase greatly as the refractive index of the film increases. This is useful for characterizing such films since the refractive index of the film can be estimated 


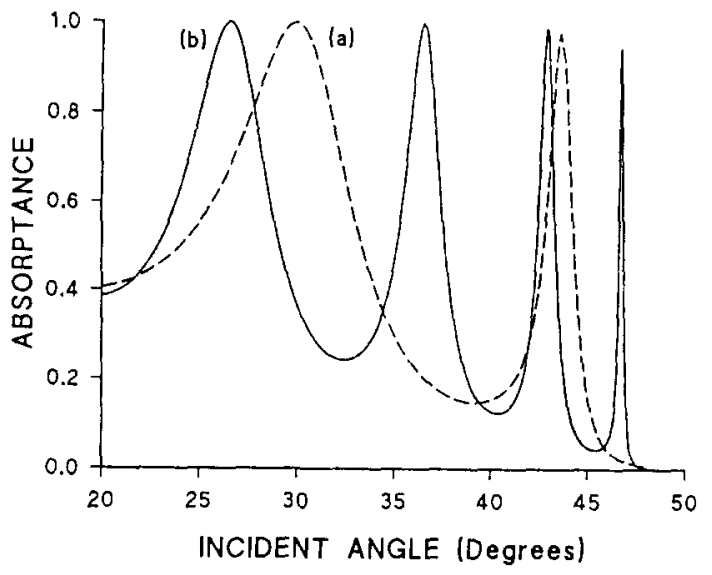

Fig. 6. Theoretical results for the angular PA spectra of thin films on a silicon substance. The refractive index of the film is 1.46 , and the thicknesses are $0.5 \mu \mathrm{m}$ (curve a) and $1.0 \mu \mathrm{m}$ tcurve b).

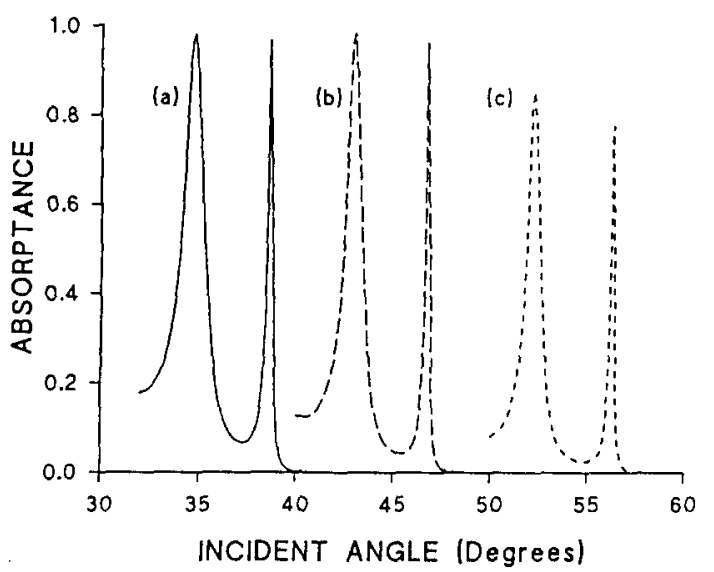

Fig. 7. Theoretical results of angular PA spectra of thin films on a silicon substrate. The thickness of the film is $1.0 \mu \mathrm{m}$, and the refractive indices are 1.26 (curve a), 1.46 (curve b) and 1.66 (curve c).

approximately from the position of the absorption peak corresponding to the largest incident angle. All the numerical results show that the positions of the resonance peaks are a function of the thickness and refractive index of the film. Therefore the thickness and/or refractive index can be determined by fitting the theoretical results to those of the experiment.

Curve d of Fig. 3 is the calculated angular PA spectrum in accordance with experimental conditions. Based on the calculation the refractive index and the thickness of the LB film are obtained as 1.49 and $1250 \AA$ respectively. In general, the LB film is very thin (about $1000 \AA$ ), and only one resonance mode, i.e. one absorption peak, exists in each film. Thus only one parameter (thickness or refractive index) can be determined by each spectrum. However, further experimental and numerical results show that, as the thickness of the LB film increases, more absorption peaks appear due to the increase of the number of the resonance modes. Then both thickness and refractive index can be easily determined simultaneously.

\section{Conclusions}

(i) The PA resonance absorption spectrum method was developed to study the optical properties and the thickness of LB films. Because the PA signal detected by a PZT piezoelectric transducer is proportional to the optical energy absorbed by the sample the wavelength and/or the incident angle of the excitation source can be changed arbitrarily over wider ranges, and neither the position nor the element of the receiver should be changed.

(ii) The refractive index of the thin film can be estimated from the position of the resonance absorption peak corresponding to the largest incident angle. By theoretical analysis of the experimental spectra, both the refractive indices and the thickness of the films can be evaluated.

(iii) The experimental resonance absorption spectra can be matched satisfactorily by the theory if the laser intensity is not very high. However, as the intensity of the laser beam increases, additional peaks appear in the angular PA spectrum, which apparently cannot be explained by the linear theory. It is predicted that the additional peaks are related to the optical non-linearity of the LB films. More information of the LB films probably may be obtained through the theoretical analysis of the additional peaks in the angular PA spectrum. Further theoretical study is in progress and will be published elsewhere.

(iv) From the description above, the similar photothermal (PT) techniques [8] can also be used to combine with ATR. However, the PT detector must follow the angular variation of the sample stage as in the conventional ATR method, except that the angular position of the pump source could be changed to satisfy the variation of the incident angle of the light. Generally, it is not so easy to move the optical source. In contrast, the PA signal can also be detected by a gas cell with a microphone [9]. In this case, the incident angle and the modulation frequency of the pump beam are limited to small angles and low frequencies respectively; then the method is not very suitable for characterizing the thin films deposited on substrates, but the disadvantages can easily be overcome with a PZT detector. Therefore the PA ATR method with a PZT detector has the advantages of a simpler optical system, higher sensitivity and wider application potential.

\section{Acknowledgment}

This work is supported by the Foundation of the National Education Commission of China. 


\section{References}

I B. Bosacchi and R. C. Oehrle, Appl. Opt., 21 (1982) 2167.

2 B. Bosacchi, R. C. Oehrle and E. Gross, Appl. Phys. Lett., 51 (1987) 158

3 A. Rosencwaig, Photoacoustics and Photoacoustic Spectroscopy, Wiley, New York, 1980.

4 Y. C. Shen and S. Y. Zhang, IEEE Trans. Ultrason. Ferroelec. Freq. Control, 39 (1992) 227.
5 S. Y. Zhang and L. Chen, in A. Mandelis (ed.), Photoacoustic and Thermal Wave Phenomena in Semiconductors, Elsevier, Amsterdam, 1987, Chapter II.

6 G. G. Roberts, Adv. Phys., 34 (1985) 475.

7 H. Kuhn, Thin Solid Films, 178 (1989) I.

$8 \mathrm{~J}$. A. Sell, Photothermal Investigation of Solids and Fluids, Academic Press, New York, 1988.

9 S. Y. Qiu, S. Y. Zhang, L. H. Wei and C. N. Hu, J. Appl. Sci., 4 (1986) 207 (in Chinese). 